\title{
Using Non-Subjective Approximation Algorithm of D-S Evidence Theory for Improving Data Fusion
}

\author{
Ning Zhang ${ }^{\mathrm{a}, \mathrm{b}}$, Peng Chen ${ }^{\mathrm{c}}$, Kai He ${ }^{\mathrm{c}}$, Zhao $\mathrm{Li}^{\mathrm{c}}$, and Xiaosheng $\mathrm{Yu}^{\mathrm{c}, *}$ \\ ${ }^{a}$ Remote Sensing Application Center, Ministry of Housing and Urban-Rural Development of the People's Republic of China \\ Beijing, 100835, China \\ ${ }^{b}$ Institute of Geographic Sciences and Natural Resources Research, CAS, Beijing, 100101, China \\ ${ }^{c}$ College of Computer and Information, Three Gorges University, Yichang, 443002, China
}

\begin{abstract}
The paper efficiently processes the issue of "focal element explosion" produced when many focal elements are fused according to D-S evidence theory. The effectiveness of subjective approximation algorithms is low since they heavily involve artificial participation. In addition, the accuracy of the results calculated by the non-subjective approximation algorithm is better. In this paper, a non-subjective approximation algorithm based on evidence levels is proposed to address the above-mentioned problem. First, the evidence level is mainly determined by the cumulative mass value of the main focal element, and the number of focal elements is reduced by approximate treatment according to the corresponding initial standard determined by the levels of evidence. Second, to further increase the accuracy of the results, the levels of evidence are used to determine the order of fusion and the discounts of evidence. It obvious that even if there is erroneous or uncertain evidence in the fused evidence, it will not affect the results significantly. The experimental results show that the algorithm outperforms others in terms of adaptability and accuracy.
\end{abstract}

Keywords: data fusion; approximation algorithm; evidence theory

(Submitted on September 17, 2019; Revised on October 16, 2019; Accepted on October 21, 2019)

(c) 2019 Totem Publisher, Inc. All rights reserved.

\section{Introduction}

The scientific judgment of fire hazards often requires the use of effective methods to fuse the uncertainties associated with information from multiple organizations. D-S evidence theory [1-2] is widely used in the fields of expert systems [3], data fusion [4-6], pattern recognition [7], decision analysis [8-10] and so on, because of its advantages in the expression and fusion of uncertain problems. When the Dempster combination rule [11] is used to deal with uncertain problems, the problem of "focal element explosion" often occurs because of the excessive number of focal elements. In order to solve this problem, researchers at home and abroad have proposed different solutions from different angles. Smets proposed a TBM double layer model [12]. The number of focal elements was reduced by using the pignistic probability to transform the compound focal element into a single focal element, but the compound focal element considered only a small part of the evidence. The numbers of the compound focal element were reduced, but the problem of "focal elements explosion" could not be solved. Dubois and Prade put forward the consonant approximation method [13]. Focal elements were nested, and the number of complex focal elements was not more than the number that the focal element used to fuse after the approximate calculation. The compound focal element obtained by the method is convenient only for description, and great errors will be generated if it is used for subsequent calculation. Although the algorithm solves the problem of "focal element explosion", the result deviates too much from reality. Voorbraak proposed a non-subjective Bayes approximation algorithm [14], which can also reduce the number of focus elements by solving the fusion problem of complex focal elements. The effect was improved remarkably for evidence with more complex focal elements, but the problem of "focal element explosion" caused by many single focal elements cannot be effectively solved. Both the $(\mathrm{k}, \mathrm{l}, \mathrm{x})$ approximate method proposed by Tessem and the summarization approximation method [15] proposed by Lowrance use the subjective judgment threshold to limit the number of retained focal elements and the size of the mass value, which can reduce the number of focal elements. The two algorithms

\footnotetext{
* Corresponding author.

E-mail address: yuxiaosheng@ctgu.edu.cn
} 
provide a good solution to "focal element explosion", but both are essentially subjective approximation methods that require human participation. Therefore, they are not universally applicable. The rough approximation method [16] was proposed by Denoeux. For the convenience of calculation, single focal elements were firstly transformed into a complex focal element through "coarsening". After that, the compound focal elements were transformed into a single focal element through "refinement". The problem of "focal element explosion" was solved, but the degree of "coarsening" and "refinement" could not be controlled well. It is easy for the calculated results to deviate from reality, so the algorithm can only be used for rough computing.

This paper proposes a non-subjective approximation algorithm based on evidential levels (ELNA) that does not require human judgment and has universal applicability in dealing with "focal element explosion". Evidential levels are mainly determined by judging the cumulative mass of the core focal element, and the number of focal elements is reduced according to the different levels of evidence, which have different initial criteria. In order to increase the accuracy of the fusion results and the computational complexity, a different discount is given to different evidential levels at the end of fusion.

\section{Literature Review}

\subsection{Evidence Theory}

DS evidence theory is considered to be an effective tool for representation and treatment of uncertainty. From the mathematical point of view, the theory was originally used to model uncertainty by Shafer. At the same time, the specific quantitative model of the theory is put forward. The DS evidence theory will be briefly reviewed in the following part; for a detailed description, refer to [1-2].

It is assumed that a collection $H$ called an identification framework is defined as follows:

$$
H=\left\{H_{1}, \cdots, H_{n}, \cdots, H_{N}\right\}
$$

It consists of $N$ mutually exclusive assumptions. From the structure, we can get $2^{N}$ propositions composed of $H$, which can be expressed as

$$
2^{H}=\left\{\varnothing,\left\{H_{1}\right\}, \cdots,\left\{H_{N}\right\},\left\{H_{1} \cup H_{2}\right\},\left\{H_{1} \cup H_{3}\right\}, \cdots, H\right\}
$$

Where $2^{H}$ is called the power set of the recognition framework and each element represents a kind of hypothesis, which is called a focal element in evidence theory. The degree of support for the hypothesis is called the basic belief assignment (BBA), denoted by $\mathrm{m} .2^{H} \rightarrow[0,1]$ meets the needs as follows:

$$
\begin{gathered}
m(\varnothing)=0 \\
\sum_{A \subseteq H} m(A)=1
\end{gathered}
$$

Where $m(A)$ represents the strength of hypothesis $A$ supported by evidence and is also called the mass value of hypothesis $A$. Based on the definition of BBA, the belief function Bel and the likelihood function $P l$ can be defined as follows:

$$
\begin{aligned}
& \operatorname{Bel}(A)=\sum_{B \subseteq A} m(B) \\
& P l(A)=\sum_{B \cap A=\varnothing} m(B)
\end{aligned}
$$

$\operatorname{Bel}(A)$ can be represented as a measurement that hypothesis $A$ is true. $P l(A)$ can be represented as a measurement that hypothesis $A$ may be true. $m(A), \operatorname{Bel}(A)$, and $P l(A)$ can be seen as three estimations of the same information: general estimation, pessimistic estimation, and optimistic estimation, respectively. The confidence interval composed of the belief function $\mathrm{Bel}$ and the likelihood function $\mathrm{Pl}$ can be more intuitive to show the degree of support of the evidence.

If the data are imperfect, such as uncertain, imprecise, or incomplete, fusion is an effective solution for obtaining more relevant information. Dempster's combination rule is the first combination rule of evidence theory and one of the most widely 
used combination rules. It is defined as follows:

Assuming that $m_{1}$ and $m_{2}$ are two independent evidence on the framework $H, 2^{H}$ is the power set, and $A$ and $B$ are the elements of the power set, then combination evidences are obtained after the two evidences are fused.

$$
m_{1,2}(C)=m_{1}(A) m_{2}(B) / K
$$

Where $K$ is the normalization constant.

$$
K=1-\sum_{A \cap B=\varnothing} m_{1}(A) m_{2}(B)
$$

In order to prevent the influence of unreliable evidence on the fusion result, a discount on the evidence of fusion is often needed.

$$
\begin{gathered}
m_{w}(A)=w m(A), \quad \forall A \subset H \\
m_{w}(H)=1-w+w m(H)
\end{gathered}
$$

Where $w$ is the discount factor, and the focal $H$ stores the mass value remaining after the discount.

\subsection{Brief Introduction to Related Research}

In order to solve the problem of "focal element explosion" in evidence theory, researchers at home and abroad have studied it from different perspectives and proposed various approximation algorithms. Among them, the most representative are the Bayesian approximation algorithm, $(K, L, X)$ approximation algorithm, and summarization approximation algorithm.

Bayesian approximation algorithm. The main idea of this algorithm is to combine evidence theory with probability theory and to transform the mass value into Bayesian probability. In general, the algorithm satisfies the following rule: the Bayesian approximation of the reliability function is equal to its fused Bayesian approximation. The rule greatly reduces the computational complexity of fusion. The algorithm is a non-subjective approximation algorithm with universal applicability. Because it assigns the mass value of the composite focal element to the single one, it reduces the uncertainty of the evidence theory and loses useful information. Compared with other approximation algorithms, there are not many advantages in the terms of treating "focal element explosion".

$(K, L, X)$ approximation algorithm. The main idea of this algorithm is to reduce the number of focal elements by selecting various thresholds in order to reduce the computational burden. From the view of application, the approximation algorithm can be suitable for almost all cases. However, the threshold is determined by subjective judgment, so different evidence must be used to reselect the thresholds. Different thresholds may lead to completely different results, which cannot be widely used due to a lack of robustness.

Summarization approximation algorithm. The main idea of this algorithm is to put the smaller mass value of the focal element into the assumed focal element $\mathrm{A} 0$, without changing the approximate treatment means on the larger mass value of the focal element. The smaller mass value of the focal elements can be eradicated, the number of focal elements is decreased, while the focal elements with larger mass value are not changed; these do not significantly impact the fusion results. However, the approximation algorithm has a similar problem as the $(K, L, X)$ algorithm: it is also necessary to subjectively determine the number of reserved focal elements, and different sources may result in completely different results because of the difference in the number of reserved elements. In addition, the method also has some problems in processing when the mass values of the focal elements are equal.

In these approximation algorithms, the Bayesian algorithm does not require artificial judgment, while the other two do.

\section{Non-Subjective Approximation Algorithm based on Evidential Levels}

In order to overcome the shortcomings of the above approximation algorithms, a non-subjective approximation algorithm based on evidential levels is proposed. Firstly, the composite focal element is transformed into single focal elements according to certain rules, and then the evidence is divided into different levels according to the cumulative mass of the 
main focal element. In order to ensure the reliability of the results, the mass values of the focal element abandoned by the approximate algorithm respectively add a certain proportion into the reserved focal elements. Finally, the evidence is fused in accordance with different weights given by the different evidence levels, which can ensure that the evidence with poor judgment will not significantly impact the results. The specific process is as follows:

\subsection{Processing Rules for Composite Focal Element}

According to the processing method of pignistic probability, the mass value of the composite focal element is allocated proportionally to all single focal elements that make up the element, so that the composite focal elements are transformed into single focal elements.

$$
M(A)=\sum_{B \subseteq H} m(B)\left(\frac{|A \cap B|}{|B|}\right), \quad \forall A \subseteq H
$$

The focal element $A$ is a subset of the focal element $B$, where $|B|$ represents the number of single focal elements contained in the composite focal element $B$, that is, $|A \cap B| /|B|$ represents the proportion of focal element $A$ to focal element $B$, and $m(A)$ is obtained from the rule.

\subsection{Divide the Different Levels of Evidence}

In order to partition the certain levels of different evidence by the cumulative mass value, the mass value of evidence is ranked in descending order after being treated in the first step. The sum of the top three focal elements and the first five focal elements are respectively recorded as $A$ and $B$, and they meet the condition $A, B \in[0,1]$. According to the cumulative mass value $A, B$ the evidential levels will be divided as follows:

$$
r(A, B)= \begin{cases}1, & A \geq 0.9 \\ 2, & A \geq 0.7 ; B \geq 0.9 \\ 3, & A<0.7 ; B \geq 0.7 \\ 4, & B<0.7\end{cases}
$$

The numbers in the formula, such as 1, 2, 3, and 4, represent the certain level of the evidence. The smaller the number, the more reliable the evidence. The evidence will be divided into four levels according to the cumulative mass values $A$ and $B$. The evidential levels of all evidence are recorded, and they will be used as the basis in the next approximation and in determining the weight ratio in the final fusion.

\subsection{Approximate Treatment}

For the convenience of subsequent calculations, all sources must be preprocessed.

Preprocessing: To retain the five focal elements with the largest mass value, abandon the other focal elements. The mass values are assigned to the five focal elements in proportion.

Approximate treatment: Different initial criteria are given according to the different levels of evidence. The mass values exceeding the initial standard are retained, and the mass values below the initial standard are abandoned.

The primary standard is set exactly according to the level of evidence. The purpose is to further reduce the number of focal elements and highlight the principal factors. The initial criteria for different levels are determined by the function $s$.

$$
s(r(A, B))= \begin{cases}\frac{1-A}{2}, & r(A, B)=1 \\ \frac{B-A}{2}, & r(A, B)=2 \\ \frac{1-B}{2}, & r(A, B)=3 \\ \frac{1}{5}, & r(A, B)=4\end{cases}
$$


The standard is confirmed by the certain levels of the initial evidence. The higher the level of evidence, the lower the standard, which is conducive to eliminate the focal elements whose mass values are small. The lower the level of evidence, the higher the standard, which is conducive to improve the certainty of the evidence after processing and facilitate the fusion of different evidence by the weight ratio in the next step.

\subsection{Data Fusion}

The purpose of approximation is to improve data fusion, but many approximate processes are likely to lose a large amount of key information. If the evidence is directly fused, the final result does not often have high credibility, so it cannot be used as a basis for decision-making.

The level of evidence is ascertained by the initial data of the evidence, which is used to determine the weight ratio of the fusion. If the level of evidence is smaller, more weight ratios can be assigned in the next fusion. If the level of evidence is higher, only its main compositions need to be reserved, so smaller weight ratios can be assigned in the next fusion. The final results of the fusion will not deviate too much from reality.

\subsubsection{Evidence Ranking based on Evidence Levels}

Reasonable fusion order is one of the effective means to obtain the correct fusion result.

Sorting in the evidence: The importance of the focus elements in the evidence is ranked according to the principle of the obedience of minority to majority. The mass values of the same focus elements in the evidence are averaged. The larger the average, the more important the focus element.

Sorting between evidences: Firstly, the evidences in different levels are sorted according to the principle that the smaller the level, the higher the priority. The evidences with smaller levels are more reliable. Integrating the evidence firstly can improve its chances of being the dominant evidence, and it is beneficial to improve the reliability of the fusion result. Secondly, the evidences in the same level are sorted according to the principle that the smaller the mass value, the higher the priority. This can effectively avoid the influence of the fusion result on the wrong evidence. Even if the wrong evidences are combined, the influence of wrong evidence on the fusion result can be minimized by using the method above.

\subsubsection{Discounting Operation}

The data processed before are fused by levels according to the different weight ratio can be obtained by the level difference between the evidence. The weight ratio in the fusion is

$$
\text { large: small }=(0.5+0.1 \times L D):(0.5-0.1 \times L D)
$$

Where the $L D$ (level difference) is the absolute value of the difference between the two evidential levels $r(A, B)$.

The evidence is fused according to its levels from small to large, and the levels of evidence after fusion are equal to the average of the two levels of evidence used to fuse. Evidence with smaller levels will reduce the levels of fused evidence, which can increase the reliability of evidence. Meanwhile, evidence with larger levels will increase the levels of fused evidence, which can reduce the reliability of evidence. The remaining mass values fused by the remaining portion of the two evidence discount are allocated according to the proportion of the dominant evidence, which is the evidence that has a smaller evidential level. If there are multiple dominant evidences, one is chosen randomly.

The selection means of dominant evidence require that there are four or more correct evidences in the same level during the fusion. This ensures that the probability of obtaining the correct result is at least $93.75 \%$. The greater the amount of nonerroneous evidence, the smaller the influence of fusing the erroneous evidence, and the higher the accuracy of the results. This can be obvious when the levels of evidence are lower. It will not have a significant impact on the fusion results when the levels of evidence are higher, even if there is some erroneous evidence.

The process for discounting and judging the levels of evidence is presented in Figure 1. 


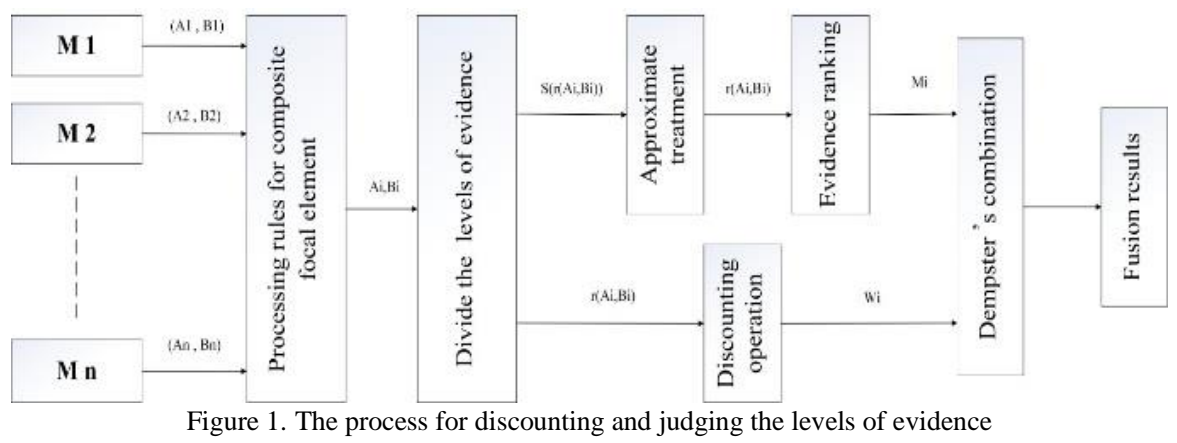

\section{Simulation Results and Analysis}

\subsection{Simulation Results and Analysis in General Cases}

In the section, the authors mainly focus on the fusion in general cases, including nested focus element evidences M1 and M2, multi-focus element evidence M4, and less discriminating focus element evidence M3. The generality of the ELNA methods and the reliability of the ELNA fusion results are verified by comparing ELNA with two other methods. For the fusion of failure evidence, the fusion of failure evidence in the two other methods will produce results that are inconsistent with the actual situation. Thus, it is useless to compare ELNA with the two other methods in the reliability. In fact, erroneous evidence only accounts for a small part of the overall evidence, so it is difficult for the direct fusion of wrong evidence to reflect the actual situation. It also cannot explain the generality of the method. Therefore, simulation results and analysis in special cases will be listed separately below.

The basic belief assignments and focal elements of the recognition framework are shown in Table 1. The focal elements are preprocessed by the approximate algorithm, non-subjective Bayesian approximation algorithm, and subjective summarization approximation algorithm. The results are shown in Tables $2-4$ The fused results were respectively obtained by the three methods, and the fusion results are shown in Table 5. In addition, if the mass value of the corresponding focal element after preprocessing is 0 , the evidence cannot be fused. Generally, the mass values of the table that are zero should be fine-tuned, that is, the corresponding disturbed value should be added according to the fact that is conducive to fusion calculation. The perturbed values are substituted by the value zero in the table. The smaller the perturbed values, the better the fusion performance. The corresponding disturbed values are usually set according to the accuracy of the data; for example, the accuracy of the data is two digits after the decimal point, so the disturbed value $\varepsilon$ is 0.01 here.

Table 1. Basic belief assignment (BBA) and focal element

\begin{tabular}{ccccccc}
\hline Focal element & $\boldsymbol{A}$ & $\boldsymbol{B}$ & $\boldsymbol{C}$ & $\boldsymbol{D}$ & $\boldsymbol{A}, \boldsymbol{B}$ & $\boldsymbol{E}$ \\
\hline M1 & 0.55 & 0.15 & 0.1 & 0.05 & 0.1 & 0.05 \\
M2 & 0.32 & 0.18 & 0.12 & 0.1 & 0.18 & 0.1 \\
M3 & 0.2 & 0.2 & 0.2 & 0.2 & 0 & 0.2 \\
M4 & 0.23 & 0.07 & 0.1 & $\ldots$ & 0 & $\ldots$ \\
\hline
\end{tabular}

The evidence M4 is evidence that includes nine focal elements, except for the focal elements $A$ and $B$. The other focal elements are single focal elements whose mass value is 0.1 .

Table 2. ELNA preprocessing results

\begin{tabular}{|c|c|c|c|c|c|}
\hline Focal element & $A$ & $B$ & $C$ & $D$ & $E$ \\
\hline M1 & 0.67 & 0.22 & 0.11 & $0(\varepsilon)$ & $0(\varepsilon)$ \\
\hline M2 & 0.51 & 0.34 & 0.15 & $0(\varepsilon)$ & $0(\varepsilon)$ \\
\hline M3 & 0.2 & 0.2 & 0.2 & 0.2 & 0.2 \\
\hline M4 & 1 & $0(\varepsilon)$ & $0(\varepsilon)$ & $0(\varepsilon)$ & $0(\varepsilon)$ \\
\hline
\end{tabular}

Table 3. Bayesian approximation algorithm preprocessing results

\begin{tabular}{cccccc}
\hline Focal element & $\boldsymbol{A}$ & $\boldsymbol{B}$ & $\boldsymbol{C}$ & $\boldsymbol{D}$ & $\boldsymbol{E}$ \\
\hline M1 & 0.59 & 0.23 & 0.09 & 0.045 & 0.045 \\
M2 & 0.42 & 0.31 & 0.1 & 0.085 & 0.085 \\
M3 & 0.2 & 0.2 & 0.2 & 0.2 & 0.2 \\
M4 & 0.23 & 0.07 & 0.1 & $\ldots$ & $\ldots$ \\
\hline
\end{tabular}


Table 4. Summarization approximation algorithm preprocessing results

\begin{tabular}{ccccccc}
\hline Focal element & $\boldsymbol{A}$ & $\boldsymbol{B}$ & $\boldsymbol{C}$ & $\boldsymbol{D}$ & $0(\varepsilon)$ & $0(\varepsilon)$ \\
M1 & 0.55 & 0.15 & $0(\varepsilon)$ & $0(\varepsilon)$ & $0(\varepsilon)$ & 0.3 \\
M2 & 0.32 & 0.18 & 0.2 & 0.2 & 0.4 \\
M3 & 0.2 & 0.2 & $0(\varepsilon)$ & $0(\varepsilon)$ & 0.2 \\
M4 & 0.23 & $0(\varepsilon)$ & $0(\varepsilon)$ & 0.77 \\
\hline
\end{tabular}

The comparison figures are shown in Figures 2-4, according to the fusion results listed in Table 5.

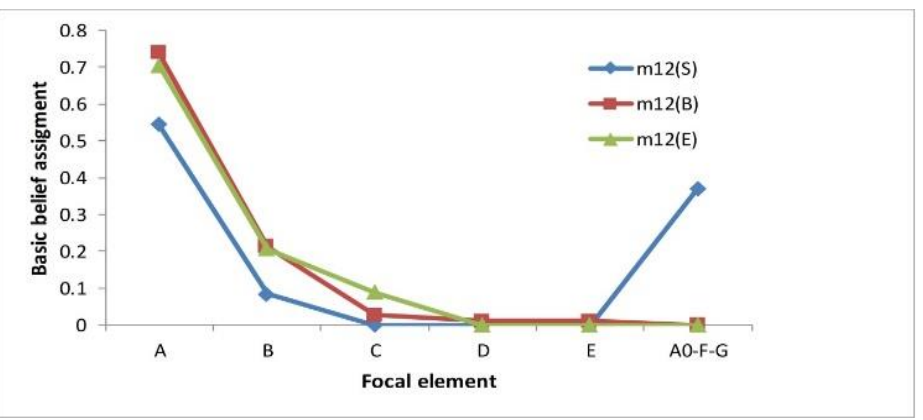

Figure 2. Fusion results of two evidences (m12)

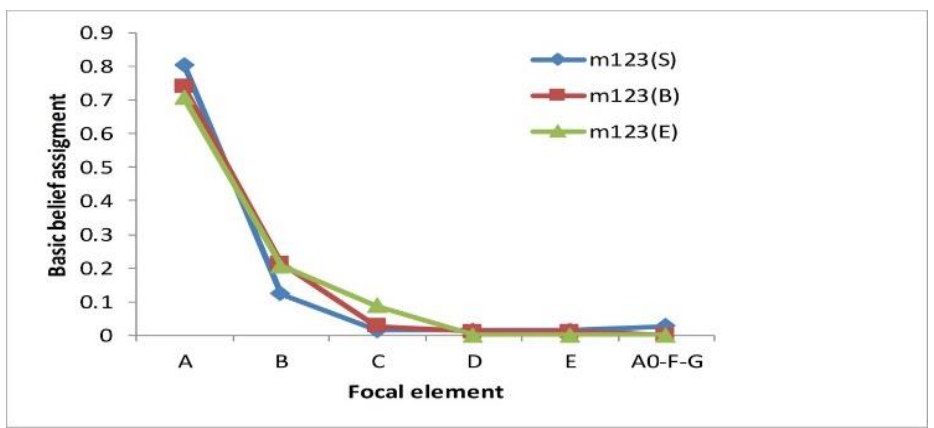

Figure 3. Fusion results of three evidences (m123)

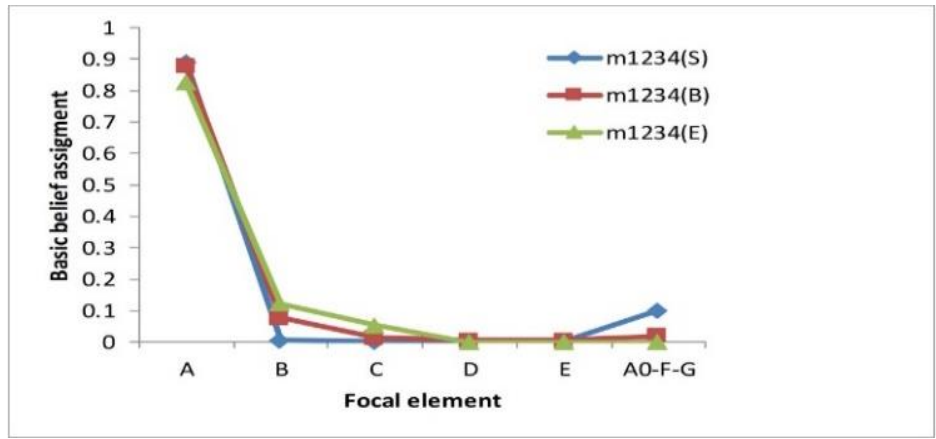

Figure 4. Fusion results of four evidences (m1234)

From the analysis of the preprocessed results, whether it is the abandonment of focal element (evidence M1, M2) with smaller mass values or the process of evidence (evidence M4) with a number of focal elements, ELNA is similar to the subjective summarization approximation algorithm and demonstrates better performance than the non-subjective Bayesian approximation algorithm in the process results. The problem of "focus element explosion" can be solved by ELNA in terms of the focal numbers that need to be calculated during fusion.

According to the fusion results shown in Table 5, the fusion results of ELNA are consistent with those fused by the Bayesian approximation algorithm. The Bayesian approximation algorithm only preprocesses the composite focal elements. Its fusion results should be consistent with those of Dempster's combination rule that have not been approximated, so the fusion results of ELNA should be believed. The fusion results are the differences between the summarization approximation algorithm and the other two algorithms. The main reason is that the fusion of the lower certainty evidence (evidence M4) has a great impact on its fusion results. The support of the evidence after three evidences are fused is $0.804,0.124,0.015$, 
$0.015,0.015$, and 0.027 , and the fusion of the fourth evidence makes the fusion results have greater deviation. Assuming that ELNA does not use the discounting operation before fusion, direct fusion will also produce erroneous results similar to the summarization approximation algorithm. To avoid this problem, the discounting operation is introduced to enable ELNA to achieve correct results while reducing computational complexity. The fused results are correct and credible by weighting before fusion, that is, a lower weight is given to the evidence (such as M3, M4), which is less reliable in the above example. When dealing with evidence with poor certainty, its main focal element will be emphasized, and its most critical information will be retained. The retained information makes all evidence more effective and avoids the loss of information in the final fusion.

Table 5. Summarization approximation algorithm preprocessing results

\begin{tabular}{|c|c|c|c|c|c|c|c|c|}
\hline Method & $\mathbf{M}$ & $\mathbf{A}$ & B & $\mathbf{C}$ & D & $\mathbf{E}$ & F-G OR A0 & Num \\
\hline \multirow{3}{*}{$\begin{array}{l}\text { Bayesian approximation } \\
\text { algorithm }\end{array}$} & $m_{12}$ & 0.738 & 0.213 & 0.027 & 0.011 & 0.011 & 0 & \multirow{3}{*}{19} \\
\hline & $m_{123}$ & 0.738 & 0.213 & 0.027 & 0.011 & 0.011 & 0 & \\
\hline & $m_{1234}$ & 0.877 & 0.077 & 0.014 & 0.006 & 0.006 & 0.020 & \\
\hline \multirow{3}{*}{$\begin{array}{c}\text { Summarization } \\
\text { approximation algorithm }\end{array}$} & $m_{12}$ & 0.545 & 0.084 & 0 & 0 & 0 & 0.371 & \multirow{3}{*}{19} \\
\hline & $m_{123}$ & 0.804 & 0.124 & 0.015 & 0.015 & 0.015 & 0.027 & \\
\hline & $m_{1234}$ & 0.891 & 0.006 & 0.001 & 0.001 & 0.001 & 0.1 & \\
\hline \multirow{3}{*}{ ELNA } & $m_{12}$ & 0.706 & 0.206 & 0.088 & 0 & 0 & 0 & \multirow{3}{*}{14} \\
\hline & $m_{123}$ & 0.706 & 0.206 & 0.088 & 0 & 0 & 0 & \\
\hline & $m_{1234}$ & 0.826 & 0.122 & 0.052 & 0 & 0 & 0 & \\
\hline \multirow{3}{*}{$\begin{array}{c}\text { ELNA } \\
\text { (not using discounting } \\
\text { operation) }\end{array}$} & $m_{12}$ & 0.789 & 0.173 & 0.038 & 0 & 0 & 0 & \multirow{3}{*}{11} \\
\hline & $m_{123}$ & 0.774 & 0.170 & 0.036 & 0.01 & 0.01 & 0 & \\
\hline & $m_{1234}$ & 0.997 & 0.002 & 0.001 & 0 & 0 & 0 & \\
\hline
\end{tabular}

The column "M" in table 5 represents the fusion result. The column "Num" in table 5 is the number of focal elements that need to be calculated in the three fusion algorithms after the approximate processing.

\subsection{Simulation Results and Analysis in Special Cases}

In the section, the authors mainly focuses on fusion in special cases (error evidence). The error evidence will be directly discarded in the summarization approximation algorithm. The fusion effects of ELNA and the Bayesian approximation algorithm are compared in special cases.

In order to facilitate calculations, five focus elements of evidence in the same level are fused. The basic belief assignments and the focal elements of the recognition framework are shown in Table 6. The selection of the dominant evidence in the ELNA approximation algorithm is random, and the mathematical expectation of the fusion result will be used as the final value. The final fusion results are shown in Table 7.

Table 6. Basic belief assignment (BBA) and focal element

\begin{tabular}{ccccc}
\hline Focal element & $\boldsymbol{A}$ & $\boldsymbol{B}$ & 0.7 \\
M5 (1) & 0.1 & 0.2 & 0.1 & 0.1 \\
M6 (4) & 0.7 & 0.2 \\
\hline
\end{tabular}

\begin{tabular}{|c|c|c|c|c|c|}
\hline Method & $\mathbf{M}$ & $A$ & $B$ & $C$ & Num \\
\hline \multirow{4}{*}{$\begin{array}{l}\text { Bayesian approximation } \\
\text { algorithm }\end{array}$} & M1 & 0.389 & 0.389 & 0.222 & \multirow{4}{*}{12} \\
\hline & M2 & 0.766 & 0.109 & 0.125 & \\
\hline & M3 & 0.937 & 0.019 & 0.044 & \\
\hline & M4 & 0.984 & 0.003 & 0.013 & \\
\hline \multirow{4}{*}{ ELNA } & M1 & 0.398 & 0.398 & 0.204 & \multirow{4}{*}{16} \\
\hline & M2 & 0.592 & 0.228 & 0.180 & \\
\hline & M3 & 0.707 & 0.137 & 0.156 & \\
\hline & M4 & 0.773 & 0.090 & 0.137 & \\
\hline
\end{tabular}

The column "M" subscript indicates the number of fusions

The analysis of fusion results in error evidence shows that the fusion results of the two algorithms are not greatly influenced if the amount of evidence is sufficient, even if there is a small amount of error evidence involved. From a computational perspective, the greater the amount of fusion evidence, the stronger the reliability of fusion results for the ELNA algorithm. For computational complexity, ELNA has obvious advantages for evidence fusion with a large number of 
focus elements, but it does not perform well when there is a small number of focus elements. As seen from the fusion results of each focal element, it is obvious that the ELNA fusion result is more in accordance with the actual cases. The focal element $\mathrm{C}$ with a smaller mass value can maintain a value of 0.137 , even if the number 0.2 is fused four times. It is more intuitive that as a main focus element, the focus element $\mathrm{C}$ conforms to the actual situation of the five evidence focus elements. In contrast, the focus element $\mathrm{C}$ is basically ignored in the Bayesian approximation algorithm after the third fusion. During the fourth fusion, the mass value of the focus element $\mathrm{C}$ is directly equal to 0.013 , and the focus element $\mathrm{B}$ is ignored (the mass value is only 0.003). Thus, it cannot reflect the actual situation.

\section{Conclusions}

In this paper, a non-subjective approximation algorithm based on evidential levels (ELNA) that does not require artificial judgement is proposed. ELNA can make the traditional evidence theory be applied in practice more widely. The levels of the evidence are divided by the cumulative mass value of the focal element, different initial criteria are set according to the different levels of evidence, and the mass values of abandoned focal elements are proportionally distributed into reserved ones, which can increase the accuracy of the evidence. Finally, the correctness of the fusion results is further ensured according to the different levels of evidence given by different weights. Regardless of whether the "focal elements explosion" problem is caused by a number of focal elements and composite focal elements or the fusion in the erroneous evidence, ELNA has a corresponding solution. ELNA has strong applicability and higher accuracy, which are advantages that allow more focal elements to be processed.

\section{Acknowledgements}

This work is supported by the National Key Research and Development Program of China (No. 2016YFC0802500) and the National Natural Science Foundation of China (No. 61272236).

\section{References}

1. A. P. Dempster, "Upper and Lower Probabilities Induced by a Multivalued Mapping," Annals of Mathematical Statistics, Vol. 38, pp. 325-340, 1967

2. G. A. Shafer, "A Mathematical Theory of Evidence," Princeton, 1976

3. M. Beynon and D. Cosker, "An Expert System for Multi-Criteria Decision Making using Dempster Shafer theory," Expert Systems with Applications, Vol. 20, pp. 357-367, 2001

4. E. Lefevre and Z. Elouedi, "How to Preserve the as an Alarm in the Combination of Belief Functions?" Decision Support Systems, Vol. 56, pp. 326-333, 2013

5. R. R. Yager, "A Measure based Approach to the Fusion of Possibilistic and Probabilistic Uncertainty," Fuzzy Optimization and Decision Making, Vol. 10, pp. 91-113, 2011

6. Z. G. Liu, Q. Pan, and J. Dezert, "A New Belief-based K-Nearest Neighbor Classification Method,” Pattern Recognition, Vol. 46, pp. 834-844, 2013

7. O. Basir and X. H. Yuan, "Engine Fault Diagnosis based on Multi-Sensor Information Fusion using Dempster-Shafer Evidence Theory," Information Fusion, Vol. 8, pp. 379-386, 2007

8. P. Elmore, F. E. Petry, and R. R. Yager, "Comparative Measures of Aggregated Uncertainty Representations," Journal of Ambient lntelligence and Humanized, Vol. 5, pp. 809-819, 2014

9. R. R. Yager, “On Prioritized Multiple Criteria Aggregation," IEEE Transactions on Systems, Man, and Cybernetics: Part B, Vol. 42, pp. 1297-1305, 2012

10. R. R. Yager, "Modeling Multi-Criteria Objective Functions using Fuzzy Measures," Information Fusion, Vol. 29, pp. 105-111, 2015

11. J. B. Yang and D. L. Xu, "Evidential Reasoning Rule for Evidence Combination," Aritificial Intellgence, Vol. 205, pp. 1-29, 2013

12. P. Smets, "Belief Functions: The Disjunctive Rule of Combination and the Generalized Bayesian Theorem," Studies in Fuzziness and Soft Computing, Vol. 219, pp. 633-664, 2008

13. D. Dubois and H. Prade, "The Legacy of 50 Years of Fuzzy Sets: A Discussion," Fuzzy Sets and Systems, Vol. 251, pp. 21-31, 2015

14. F. Voorbraak, "A Computationally Efficient Approximation of Dempster-Shafer theory," International Journal of ManMachine Studies, Vol. 30, pp. 525-536, 1989

15. J. D. Lowrance and T. M. Strat, "A Framework for Evidential-Reasoning Systems," Studies in Fuzziness and Soft Computing, Vol. 219, pp. 419-434, 2008

16. T. Denoeux, "Conjunctive and Disjunctive Combination of Belief Functions Induced by Nondistinct Bodies of Evidence," Artificial Intelligence, Vol. 172, pp. 234-264, 2008 